\title{
A parametric frequency analysis for functionally graded cylinders using graded harmonic FEM
}

\author{
A.I. Karakas*iD, A. Daloglu iD \\ Karadeniz Technical University, Department of Civil Engineering, Trabzon, Turkey
}

\begin{abstract}
A graded harmonic solid ring finite element model (FEM) is developed from the three-dimensional theory of elasticity using Fourier series expansion in circumferential direction to investigate free vibration characteristics of functionally graded (FG) thin and thick-walled cylinders parametrically. The mechanical properties of the finite length FG cylinders composed of metal (stainless steel) and ceramic (silicon nitride) are assumed to vary in radial direction according to a power law variation as a function of the volume fractions of the constituents. The frequency characteristics of the FG cylinders depending on various parameters such as circumferential harmonic number, power law exponent, thickness to radius ratio, length to radius ratio, and constituent material configuration are investigated through numerical simulations. When the graded harmonic model is compared with the previous models in the literature the agreements are found to be excellent. Also, a reduction in computational effort is provided using a smaller number of graded elements required for a fair estimation of vibrational behavior of such axisymmetric structures. As far as the numerical results are considered it is observed that thin and thick-walled cylinders behave in a different way for increasing circumferential harmonic number. The influence of the power law exponent on the frequency depends on the constituent material position and it does not affect the circumferential harmonic number at which the fundamental natural frequency occurs. As a conclusion, it can be stated that the free vibration behavior of FG cylinders can be regulated arbitrarily by altering material configuration and power law function as well as geometrical parameters.
\end{abstract}

\section{Keywords}

Graded harmonic finite element; Functionally graded material; Thick hollow cylinder; Free vibration

Received: 21 October 2019; Accepted: 01 November 2019

ISSN: 2630-5763 (online) (C) 2019 Golden Light Publishing All rights reserved.

\section{Introduction}

Classical layered composite materials, thanks to a combination of superior properties of different materials such as metals and ceramics, have been widely used for various engineering applications. However, in classical layered composite, there may exist sort of stress concentrations at the interface where two different material layers meet, which can produce undesired results. To overcome such defects studies on the development of new layered composites, functionally gradient material (FGM), have been intensively progressed since late 1980's. FGM has a material composition varying continuously (without any discontinuity) through the thickness. These kinds of materials are anisotropic and inhomogeneous with continuous variation in mechanical properties. A cylindrical structure made up of such a composite material,

\footnotetext{
Corresponding author

Email: aliihsan.karakas@ktu.edu.tr
} 
FGM, is one of the basic structural configurations because of its simple geometrical shape [1]. Studies on vibration of cylinders are extensive. While Ludwig and Krieg [2], Chung [3], Bhimaraddi [4] concentrated on the vibration of thin-walled cylinders, Wong and Williams [5], Loy and Lam [6], and Singhal et al. [7] studied the vibration of thick-walled cylinders. But they are all limited to isotropic cylinders.

Several studies on FGM cylindrical structures have also been carried out. Loy et al. [8] obtained the natural frequencies of simply supported FGM cylindrical shells by using Love's shell theory and the Rayleigh-Ritz method.In a similar work, Pradhan et al. [9] studied the natural frequencies of FGM cylindrical shells under various boundary conditions. Kadali and Ganesan [10] simulated thermal buckling and free vibration of FGM cylinders using first order shear deformation theory and Fourier series expansion of displacement field in circumferential direction. Iqbal et al. [11] applied wave propagation approach to analyze vibration of circular cylindrical shells with functionally graded material.

Arshad et al. [12] performed an associative study for natural frequencies of two-layered cylindrical shells that was presented with one layer made up of functionally graded material and the other layer of isotropic material. Shah et al. [13] presented an analysis on the vibrations of FG cylindrical shells founded on the Winkler and Pasternak foundations. Naeem et al. [14] studied the vibration frequency characteristics of FG cylindrical shells using the generalized differential quadrature method. Ansari et al. [15] presented an analytical solution for free vibrations of FGM cylinders using first order shear deformation theory. The free vibration characteristics of functionally graded material elliptical cylindrical shells using finite element procedure are studied using higher order displacement model including variable transverse displacement through the thickness by Patel et al. [16]. Haddadpour et al. [17] analyzed free vibrations of simply supported FGM cylinders with thermal dependent material properties by the Galerkin method. All of these studies focused on functionally graded thin walled cylinders. The study of free vibrations of thick functionally graded hollow circular cylinder using three dimensional theory of elasticity is relatively scarce in the literature compare to the study of thin functionally graded cylindrical shells using shell theories. For the analysis of functionally graded thick hollow cylinders conventional finite element formulations assign a single set of material properties to each element such that the property field is constant within an individual element. To model a continuously nonhomogeneous material one must discretize the material property functions at the size scale of the element mesh, producing a step-wise constant approximation to the property field. This step-wise constant approximation to continuous material properties has been widely used in the literature involving numerical simulations of functionally graded materials [18, 19]. Additionally, a graded finite element formulation can be used to analyze such thick FG cylinders. For the conventional finite element formulation, the modulus of elasticity at each element centroid was used as the element stiffness. For the graded formulation, the local modulus at each integration point was used. Santare et al. [20], Kim and Paulino [21] showed that graded finite elements have the potential to provide improved accuracy without increasing the number of degrees of freedom for a given model. Taghvaeipour et al. [22] developed a new cylindrical element with graded finite element formulation to analyze FGM hollow cylinders.

The purpose of the current work is to develop a harmonic finite element model for the free vibration analysis of functionally graded thin and thick hollow cylinders with varying structural properties in the radial direction on the basis of threedimensional theory of elasticity. In this paper the graded harmonic quadrilateral ring element with nine nodes and three displacement degrees of freedom per node is developed and the procedure of extracting the element stiffness and mass matrices is completely illustrated. Several functionally graded cylinders with different power law exponents, constituent material configurations and geometrical dimensions are investigated for 
frequency characteristics using these graded matrices. The resulted natural frequencies are compared against previous studies in the literature and presented in tabular and graphical formats.

\section{Material properties in FGM cylinders}

The functionally graded material (FGM) composes of two different materials usually ceramic and metal alloy, the mixing ratio of which is varied spatially continuously and gradually. For a composite material consists of two phases, the effective value of a material property $(P)$ of the composite is computed based on the rule of mixtures by Darabseh [23]:

$$
P=P_{m} V_{m}+P_{c} V_{c}
$$

where $V_{m}$ and $V_{c}$ are the volume fractions of the metal and ceramic, respectively. The volume fractions satisfy the relation $V_{m}+V_{c}=1$. The volume fraction of the metal $V_{m}$ using the power law is defined as

$V_{m}=V_{m, i}+\left(V_{m, o}-V_{m, i}\right)\left(\frac{r-r_{i}}{r_{o}-r_{i}}\right)^{S}$

where $r_{i}$ is the inner radius of the cylinder, $r_{o}$ is the outer radius of the cylinder, $V_{m, i}$ and $V_{m, o}$ are the volume fractions of the metal constituent on the inner and outer surfaces, respectively, and $S$ is the power law exponent that represents the graded distribution along the radial direction. The cylinder has a linear variation for $S=1$. As the parameter $S$ increases, the cylinder becomes rich in ceramic when $V_{m, i}=0$ and becomes rich in metal when $V_{m, i}=1$. Conversely, as $S$ decreases, the cylinder becomes rich in metal when $V_{m, i}=0$ and becomes rich in ceramic when $V_{m, i}=1$.

The properties of FGM are calculated by substituting the volume fractions of the material constituents into the rule of mixtures. Some of properties are expressed as follows (Darabseh 2011).

$$
\begin{aligned}
& E(r)=E_{m}\left(\left(1-\frac{E_{c}}{E_{m}}\right) V_{m}+\frac{E_{c}}{E_{m}}\right) \\
& v(r)=v_{m}\left(\left(1-\frac{v_{c}}{v_{m}}\right) V_{m}+\frac{v_{c}}{v_{m}}\right) \\
& \rho(r)=\rho_{m}\left(\left(1-\frac{\rho_{c}}{\rho_{m}}\right) V_{m}+\frac{\rho_{c}}{\rho_{m}}\right)
\end{aligned}
$$

where $E(r), v(r)$, and $\rho(r)$ are radially varying Young's modulus, Poisson's ratio and mass density, respectively. The change of Young's modulus throughout the wall thickness when the volume fractions of the metal constituent on the inner and outer surfaces are 0 and 1 , respectively, is shown in Fig. 1.

The rate of decrease of modulus of elasticity for $S<1$ is high compared to $S>1$ at radius closer to the inner surface and the rate of decrease of modulus of elasticity for $S>1$ is much higher than for $S<1$ at radius closer to the outer surface.

\section{Harmonic finite element model}

For the harmonic model, the three-dimensional elasticity theory is used and the equations of motion are obtained by expanding the displacement field in the Fourier series in terms of the circumferential coordinate $\theta$ as:

$$
\{u\}=\left\{\begin{array}{l}
u_{r}(r, \theta, z) \\
u_{\theta}(r, \theta, z) \\
u_{z}(r, \theta, z)
\end{array}\right\}=\left\{\begin{array}{l}
\sum_{m=0}^{\infty} \bar{u}_{r m} \cos m \theta+\sum_{m=0}^{\infty} \overline{u_{r m}} \sin m \theta \\
\sum_{m=0}^{\infty} \bar{u}_{\theta m} \sin m \theta-\sum_{m=0}^{\infty}=\overline{u_{\theta m} \cos m \theta} \\
\sum_{m=0}^{\infty} \bar{u}_{z m} \cos m \theta+\sum_{m=0}^{\infty} \overline{u_{z m}} \sin m \theta
\end{array}\right\}
$$

where $m$ is the circumferential mode (harmonic) number and symbols $u_{r}, u_{\theta}$ and $u_{z}$ indicate the radial, circumferential and axial displacement components, respectively, see Fig. 2. All barred quantities in Eq. (6) are amplitudes approximated using the finite element method, which are functions of $r, z$ but not of $\theta$. This leads to a harmonic finite element in the $(r, z)$ plane. 


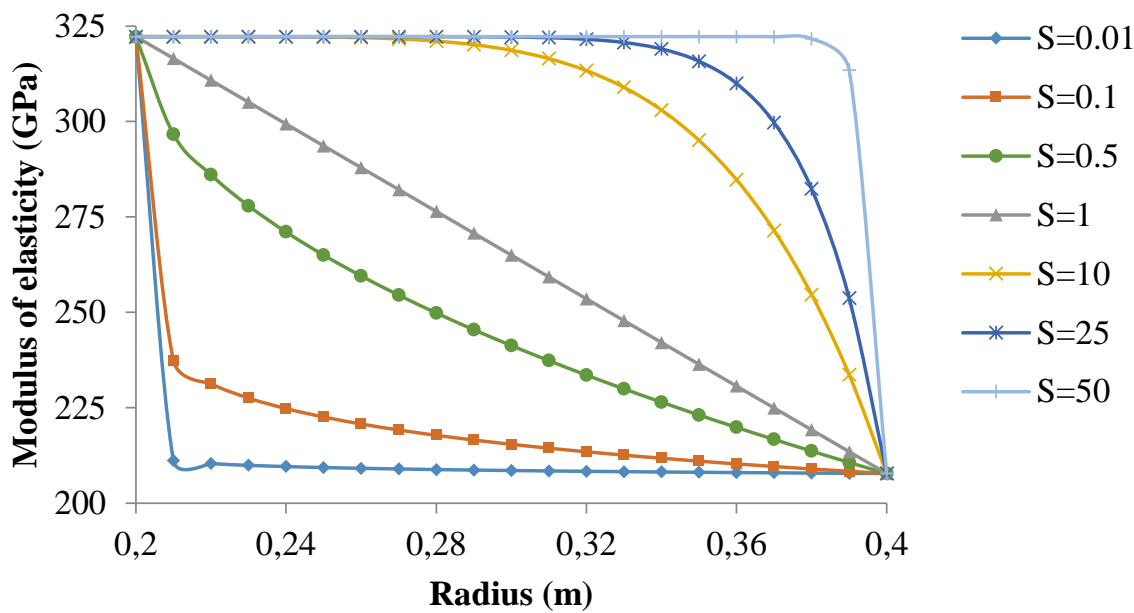

Fig. 1. Distribution of modulus of elasticity throughout radius

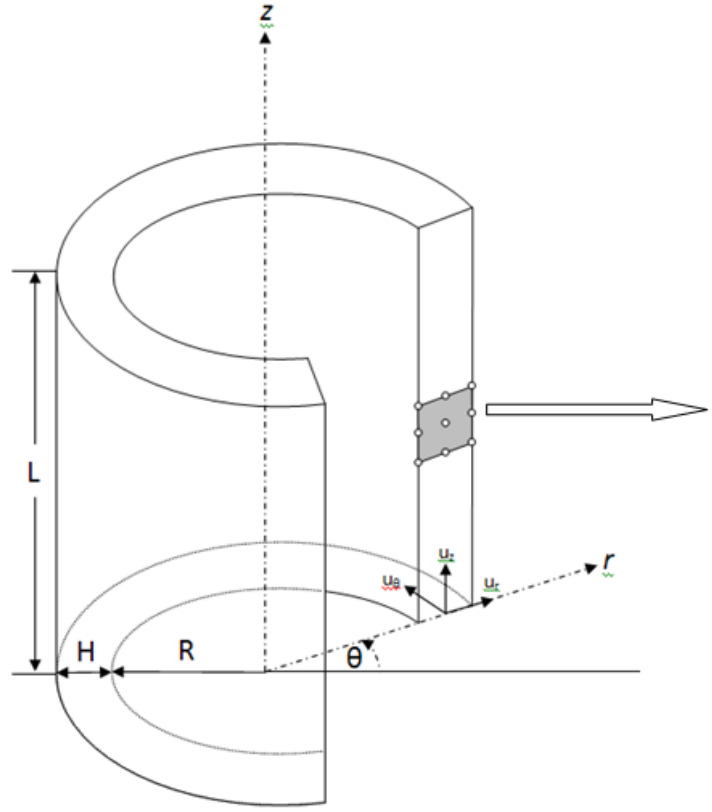

(a)

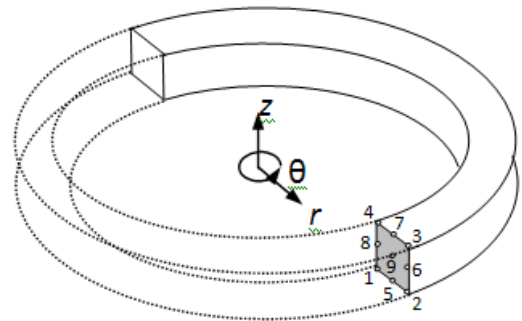

(b)

Fig. 2. (a) Displacement components in cylindrical reference system (b) a solid ring finite element

Single barred amplitudes represent symmetric displacement components (displacements which have $\theta=0$ as a plane of symmetry), while double barred amplitudes represent anti-symmetric displacement terms. The amplitudes of the displacement components in Eq. (6) can be interpolated from the corresponding nodal amplitudes using the shape functions for a finite element.

Therefore, the vector of displacement field within the element for the Fourier term $m$ can be described in the following form [24]: 


$$
\begin{aligned}
&\{u\}_{m}=\left\{\begin{array}{l}
u_{r m}(r, \theta, z) \\
u_{\theta m}(r, \theta, z) \\
u_{z m}(r, \theta, z)
\end{array}\right\} \\
&=\left[\overline{g_{\theta m}}\right]_{u}[N]\left\{\bar{d}_{m}\right\}+\left[\overline{\overline{g_{\theta m}}}\right]_{u}[N]\left\{\overline{\bar{d}}_{m}\right\} \\
& {\left[\overline{g_{\theta m}}\right]_{u}=\left[\begin{array}{ccc}
\cos m \theta & 0 & 0 \\
0 & \sin m \theta & 0 \\
0 & 0 & \cos m \theta
\end{array}\right] }
\end{aligned}
$$

and

$$
\left[\overline{\overline{g_{\theta m}}}\right]_{u}=\left[\begin{array}{ccc}
\sin m \theta & 0 & 0 \\
0 & -\cos m \theta & 0 \\
0 & 0 & \sin m \theta
\end{array}\right]
$$

Strains and stresses in an element can also be stated in terms of the Fourier series. For a Fourier term $m$, the strain vector in cylindrical coordinates can be written as,

$$
\begin{aligned}
& \left\{\varepsilon_{m}\right\}=\left\{\begin{array}{l}
\bar{\varepsilon}_{r m} \\
\bar{\varepsilon}_{\theta m} \\
\bar{\varepsilon}_{z m} \\
\bar{\gamma}_{r z m} \\
\bar{\gamma}_{r \theta m} \\
\bar{\gamma}_{\theta z m}
\end{array}\right\}+\left\{\begin{array}{l}
\overline{\bar{\varepsilon}}_{r m} \\
\overline{\bar{\varepsilon}}_{\theta m} \\
\overline{\bar{\varepsilon}}_{z m} \\
\overline{\bar{\gamma}}_{r z m} \\
\overline{\bar{\gamma}}_{r \theta m} \\
\overline{\bar{\gamma}}_{\theta z m}
\end{array}\right\} \\
& \begin{array}{c}
\left\{\begin{array}{c}
\frac{\partial u_{r m}}{\partial r} \\
\frac{1}{r}\left(u_{r m}+\frac{\partial u_{\theta m}}{\partial \theta}\right) \\
\frac{\partial u_{z m}}{\partial z} \\
\frac{\partial u_{z m}}{\partial r}+\frac{\partial u_{r m}}{\partial z} \\
\frac{1}{r} \frac{\partial u_{r m}}{\partial \theta}+\frac{\partial u_{\theta m}}{\partial r}-\frac{u_{\theta m}}{r} \\
\frac{\partial u_{\theta m}}{\partial z}+\frac{1}{r} \frac{\partial u_{z m}}{\partial \theta}
\end{array}\right\} \\
=\left(\left[\bar{B}_{m}\right]\left\{\bar{d}_{m}\right\}+\left[\overline{\bar{B}}_{m}\right]\left\{\overline{\bar{d}}_{m}\right\}\right)
\end{array}
\end{aligned}
$$

where $\left[\bar{B}_{m}\right]$ and $\left[\bar{B}_{m}\right]$ are the matrices which relate the symmetric and anti-symmetric nodal displacement amplitudes with corresponding strains. The strain-nodal displacement matrix for $i^{\text {th }}$ node and symmetric Fourier terms is obtained as:

$$
\left[\bar{B}_{i m}\right]=\left[\begin{array}{ccc}
N_{i, r} \cos m \theta & 0 & 0 \\
\frac{N_{i}}{r} \cos m \theta & \frac{m N_{i}}{r} \cos m \theta & 0 \\
0 & 0 & N_{i, z} \cos m \theta \\
N_{i, z} \cos m \theta & 0 & N_{i, r} \cos m \theta \\
-\frac{m N_{i}}{r} \sin m \theta & \left(N_{i, r}-\frac{N_{i}}{r}\right) \sin m \theta & 0 \\
0 & N_{i, z} \sin m \theta & -\frac{m N_{i}}{r} \sin m \theta
\end{array}\right]
$$

Similarly, $\left[\overline{\bar{B}}_{i m}\right]$ can be obtained from that of $\left[\bar{B}_{i m}\right]$ by simply substituting $-\sin m \theta$ with $\cos m \theta$ and $\cos m \theta$ with $\sin m \theta$.

The constitutive equation for FGM is written for $m^{\text {th }}$ harmonic as:

$$
\left\{\sigma_{m}\right\}=[D(r)]\left\{\varepsilon_{m}\right\}
$$

where the coefficients of elasticity matrix $D(r)$ is calculated as given by Bhatti [25] for a threedimensional isotropic material by substituting $E=E(r)$ for Young's modulus and $v=v(\mathrm{r})$ for Poisson's ratio both depending on radial coordinate as given in Eqs. (3) and (4), respectively. Traditionally, the components of $D(r)$ matrix are taken to have constant material properties for each finite element. However, there is no fundamental reason that these elastic properties cannot be spatially variable functions within an element. Therefore, the components of the elasticity matrix are calculated at each Gauss point in the element for the graded harmonic finite element model.

\section{Graded harmonic finite element matrices}

\subsection{Formulation of the element stiffness matrix}

The stiffness matrix of a linear system is calculated from the derivation of the strain energy of the 
element as explained by Bhatti [25]. So, two stiffness matrices $\left[\bar{k}_{m}\right]$ and $\left[\bar{k}_{m}\right]$ defined for both single and double barred terms in Fourier series expansion are expressed as:

$\left[\bar{k}_{m}\right]=\iint_{A_{e}}\left[B_{m}\right]^{T}\left(\int_{0}^{2 \pi}\left[\left[\overline{g_{\theta m}}\right]^{T}[D(r)]\left[\overline{g_{\theta m}}\right]\right] d \theta\right)\left[B_{m}\right] r d r d z$

$\left[\overline{g_{\theta m}}\right]=\left[\begin{array}{cccccc}\cos m \theta & 0 & 0 & 0 & 0 & 0 \\ 0 & \cos m \theta & 0 & 0 & 0 & 0 \\ 0 & 0 & \cos m \theta & 0 & 0 & 0 \\ 0 & 0 & 0 & \cos m \theta & 0 & 0 \\ 0 & 0 & 0 & 0 & \sin m \theta & 0 \\ 0 & 0 & 0 & 0 & 0 & \sin m \theta\end{array}\right]$

$\left[\overline{\bar{k}_{m}}\right]=\iint_{A_{e}}\left[B_{m}\right]^{T}\left(\int_{0}^{2 \pi}\left[\left[\overline{\overline{g_{\theta m}}}\right]^{T}[D(r)]\left[\overline{\overline{g_{\theta m}}}\right]\right] d \theta\right)\left[B_{m}\right] r d r d z$

where the matrices $\left[\overline{g_{\theta m}}\right]$ and $\left[\overline{\overline{g_{\theta m}}}\right]$ of the harmonic functions for the harmonic $m$ are:

and

$\left[\overline{\overline{g_{\theta m}}}\right]=\left[\begin{array}{cccccc}\sin m \theta & 0 & 0 & 0 & 0 & 0 \\ 0 & \sin m \theta & 0 & 0 & 0 & 0 \\ 0 & 0 & \sin m \theta & 0 & 0 & 0 \\ 0 & 0 & 0 & \sin m \theta & 0 & 0 \\ 0 & 0 & 0 & 0 & -\cos m \theta & 0 \\ 0 & 0 & 0 & 0 & 0 & -\cos m \theta\end{array}\right]$

$\left[\bar{B}_{m}\right]=\left[\overline{g_{\theta m}}\right]\left[B_{m}\right]$

$\left[\overline{\bar{B}}_{m}\right]=\left[\overline{\overline{g_{\theta m}}}\right]\left[B_{m}\right]$

It can be observed that each term in the products of $\left[\overline{g_{\theta m}}\right]^{T}[D(r)]\left[\overline{g_{\theta m}}\right],\left[\overline{\overline{g_{\theta m}}}\right]^{T}[D(r)]\left[\overline{\overline{g_{\theta m}}}\right]$ will be a function of $(E, v)$ multiplied by either $\cos ^{2} m \theta$ or $\sin ^{2} m \theta$. Thus, integration over the circumferential direction $\theta$ can be carried out explicitly using the orthogonality property of trigonometric functions and so:

$\int_{0}^{2 \pi}\left[\overline{g_{\theta m}}\right]^{T}[D(r)]\left[\overline{g_{\theta m}}\right] d \theta=\pi[D(r)]$ if $m>0(19)$

$\int_{0}^{2 \pi}\left[\overline{\overline{g_{\theta m}}}\right]^{T}[D(r)]\left[\overline{\overline{g_{\theta m}}}\right] d \theta=\pi[D(r)]$ if $m>0$ (20)

$$
\begin{aligned}
\int_{0}^{2 \pi}\left[\overline{g_{\theta m}}\right]^{T}[D(r)]\left[\overline{g_{\theta m}}\right] d \theta \\
=2 \pi\left[\begin{array}{lllllll}
1 & 0 & 0 & 0 & 0 & 0 \\
0 & 1 & 0 & 0 & 0 & 0 \\
0 & 0 & 1 & 0 & 0 & 0 \\
0 & 0 & 0 & 1 & 0 & 0 \\
0 & 0 & 0 & 0 & 0 & 0 \\
0 & 0 & 0 & 0 & 0 & 0
\end{array}\right][D(r)] \\
=2 \pi\left[\bar{D}_{0}(r)\right] \text { if } m=0
\end{aligned}
$$

$$
\int_{0}^{2 \pi}\left[\overline{\overline{g_{\theta m}}}\right]^{T}[D(r)]\left[\overline{\overline{g_{\theta m}}}\right] d \theta
$$

$$
\begin{aligned}
& =2 \pi\left[\begin{array}{llllll}
0 & 0 & 0 & 0 & 0 & 0 \\
0 & 0 & 0 & 0 & 0 & 0 \\
0 & 0 & 0 & 0 & 0 & 0 \\
0 & 0 & 0 & 0 & 0 & 0 \\
0 & 0 & 0 & 0 & 1 & 0 \\
0 & 0 & 0 & 0 & 0 & 1
\end{array}\right][D(r)] \\
& =2 \pi\left[\overline{\bar{D}}_{0}(r)\right] \text { if } m=0
\end{aligned}
$$


The integration results in a factor $\pi$ that multiplies each term for each Fourier series harmonics except for zeroth $(m=0)$ harmonic. It should also be mentioned that, due to choice of negative sign in the second expression in Eq. (6), the stiffness matrix for double barred terms is identical to that of single barred terms, that is $\left[\bar{k}_{m}\right]=\left[\bar{k}_{m}\right]=\left[k_{m}\right]$ for $m>0$. Additionally, $\left[\bar{k}_{0}\right]$ and $\left[\overline{\bar{k}}_{0}\right]$ can be used for special cases of plane axisymmetric and plane axi-antisymmetric cases respectively. So, for the $m^{\text {th }}$ harmonic the graded element stiffness matrices are obtained from the following expressions as:

$\left[\bar{k}_{0}\right]=2 \pi \iint_{A_{e}}\left(\left[B_{0}\right]^{T}\left[\bar{D}_{0}(r)\right]\left[B_{0}\right]\right) r d r d z$ if $m=0(23)$

$\left[\overline{\bar{k}}_{0}\right]=2 \pi \iint_{A_{e}}\left(\left[B_{0}\right]^{T}\left[\overline{\bar{D}}_{0}(r)\right]\left[B_{0}\right]\right) r d r d z$ if $m=0$

$\left[k_{m}\right]=\pi \iint_{A_{e}}\left(\left[B_{m}\right]^{T}[D(r)]\left[B_{m}\right]\right) r d r d z$ if $m>0$

Gauss quadrature is used for numerical integration by taking 3 points in each direction.

\subsection{Formulation of the element mass matrix}

For a functionally graded elastic material, the mass density will in general be a function of position as well as the modulus of elasticity. Therefore, in the most general graded element, the mass density distribution should be incorporated in the same manner as the modulus of elasticity and Poisson's ratio. The consistent mass matrix of an element is calculated from the derivation of the kinetic energy as explained by Bhatti [25]. The graded element mass matrices are obtained as the followings as in the case of stiffness matrix expressions

$$
\left[\bar{m}_{0}\right]=2 \pi \iint_{A_{e}} \rho(r)[N]^{T}\left[\begin{array}{lll}
1 & 0 & 0 \\
0 & 0 & 0 \\
0 & 0 & 1
\end{array}\right][N] r d r d z \text { if } m=0
$$

$$
\begin{aligned}
& {\left[\begin{array}{l}
\left.\overline{m_{0}}\right] \\
\end{array}=2 \pi \iint_{A_{e}} \rho(r)[N]^{T}\left[\begin{array}{lll}
0 & 0 & 0 \\
0 & 1 & 0 \\
0 & 0 & 0
\end{array}\right][N] r d r d z \text { if } m=0\right.} \\
& {\left[m_{m}\right]=\pi \iint_{A_{e}} \rho(r)[N]^{T}[N] r d r d z \text { if } m>0}
\end{aligned}
$$

where $\rho(r)$ is density distribution as given in Eq. (5). The result is a fully populated mass matrix that incorporates the nonhomogeneous density distribution. Three-point Gauss quadrature is used for the numerical integration.

\section{Free vibration analysis}

The equation of motion for free and undamped multi-degree of freedom system vibrations is of the form [25]:

$$
[M]\{\ddot{u}\}+[K]\{u\}=0
$$

where $\{u\}$ is the global degree of freedom vector and $[M]$ and $[K]$ are the mass and stiffness matrices, respectively. When vibrating in one of the mode shapes all points in the system undergo simple harmonic motion with the corresponding natural frequency $\omega_{i}$, which can be stated as

$$
u=\varphi_{i} \sin \left(\omega_{i} t\right)
$$

in which $\varphi_{i}$ is the nodal amplitude vector (or mode shape) with each component corresponding to the specific degree of freedom. Substituting Eq. (30) into Eq. (29) allows cancelation of the sine term which leaves

$$
\left(K-\omega_{i}^{2} M\right) \varphi_{i}=0
$$

This is the basic statement of the free vibration problem. The determinant of the coefficient matrix $\left(K-\omega_{i}^{2} M\right)$ should vanish to avoid a nontrivial solution

$$
\operatorname{det}\left([K]-\omega^{2}[M]\right)=0
$$

Solving Eq. (32) leads the square of natural angular frequencies as the eigenvalues of the generalized problem. 


\section{Numerical examples}

The natural frequencies of a simply-simply supported (S-S) thin walled functionally graded cylinder are obtained first to compare the results of the graded harmonic quadrilateral ring finite element model with the ones obtained by Loy et al. [8] for various circumferential harmonic numbers $m$ and power law exponents $S$. The results obtained in the study are presented in Table 1 along with geometric and material properties of the problem. The first axial mode is considered only, and very good agreement is obtained with the results given in the literature as can be seen in Table 1.

Similarly, for the verification of the present model for thick-walled cylinders the natural frequency for the first bending mode and torsional mode of clamped-clamped functionally graded cylinders with varying $H / R$ ratios are compared with the results obtained by Taghvaeipour et al. [22] using conventional brick elements in ANSYS and the new cylindrical superelements. The conventional finite element results are gained with sufficient number of brick elements to assure a mesh-independent result by Taghvaeipour et al. [22]. The natural frequencies are presented in Table 2 for each model. As far as the present graded harmonic model and the superelement model is compared with respect to the brick element model it can be concluded that the harmonic model requires very few elements to provide a good accuracy. Also, it should be noted that the number of harmonic elements needs to be increased considerably for acceptable results due to the nonlinear shape of the bending mode.

After the verification of the proposed graded harmonic model, a number of various thin and thick functionally graded cylinders are analyzed to investigate frequency characteristics. The FGM cylinders considered in this paper are made of silicon nitride $\left(\mathrm{Si}_{3} \mathrm{~N}_{4}\right)$ and stainless steel (SUS304). The mechanical properties of these materials at room temperature are given in Table 3.

According to constituent material configuration two different simply-simply supported cylinder models are considered. The model with $\mathrm{V}_{\mathrm{m}, \mathrm{i}}=0$ and $\mathrm{V}_{\mathrm{m}, \mathrm{o}}=1$ is called as "Model 1" and the model with $\mathrm{V}_{\mathrm{m}, \mathrm{i}}=1$ and $\mathrm{V}_{\mathrm{m}, \mathrm{o}}=0$ is called as "Model 2". In other words, the inner material of the Model 1 is silicon nitride and the outer material is stainless steel while the inner material of the Model 2 is stainless steel and the outer material is silicon nitride. The frequency results of Model ü1 are presented in tabular format whereas those of Model 2 are presented in graphical format.

Table 1. Comparison of natural frequencies (Hz) for S-S thin walled FG cylinder $(H=0.002 \mathrm{~m} . H / R=0.002, L / R=20$, $\left.E_{o}=2.07788 \times 10^{11} \mathrm{~N} / \mathrm{m}^{2}, v_{o}=0.317756, \rho_{o}=8166 \mathrm{~kg} / \mathrm{m}^{3}, E_{i}=2.05098 \times 10^{11} \mathrm{~N} / \mathrm{m}^{2}, v_{i}=0.31, \rho_{i}=8900 \mathrm{~kg} / \mathrm{m}^{3}\right)$

\begin{tabular}{cccccccccc}
\hline & \multicolumn{3}{c}{$S=0.7$} & \multicolumn{3}{c}{$S=2.0$} & \multicolumn{3}{c}{$S=30$} \\
\cline { 2 - 9 } & $\begin{array}{c}\text { Loy et al. } \\
(1999)\end{array}$ & Present & Diff.(\%) & $\begin{array}{c}\text { Loy et al. } \\
(1999)\end{array}$ & Present & Diff.(\%) & $\begin{array}{c}\text { Loy et al. } \\
(1999)\end{array}$ & Present & Diff.(\%) \\
\hline 1 & 13.269 & 13.283 & -0.11 & 13.103 & 13.117 & -0.11 & 12.914 & 12.927 & -0.10 \\
2 & 4.4994 & 4.5039 & -0.10 & 4.4435 & 4.4474 & -0.09 & 4.3765 & 4.3799 & -0.08 \\
3 & 4.1749 & 4.1697 & 0.12 & 4.1235 & 4.1186 & 0.12 & 4.0576 & 4.0525 & 0.13 \\
4 & 7.0691 & 7.0554 & 0.19 & 6.9820 & 6.9684 & 0.19 & 6.8726 & 6.8591 & 0.20 \\
5 & 11.290 & 11.267 & 0.20 & 11.151 & 11.128 & 0.21 & 10.978 & 10.956 & 0.20 \\
6 & 16.527 & 16.494 & 0.20 & 16.323 & 16.289 & 0.21 & 16.071 & 16.038 & 0.21 \\
7 & 22.735 & 22.688 & 0.21 & 22.454 & 22.408 & 0.20 & 22.108 & 22.062 & 0.21 \\
8 & 29.903 & 29.841 & 0.21 & 29.533 & 29.472 & 0.21 & 29.078 & 29.018 & 0.21 \\
9 & 38.028 & 37.950 & 0.21 & 37.559 & 37.481 & 0.21 & 36.981 & 36.904 & 0.21 \\
10 & 47.111 & 47.014 & 0.21 & 46.529 & 46.432 & 0.21 & 45.813 & 45.718 & 0.21 \\
\hline
\end{tabular}


Table 2. Comparison of natural frequencies $(\mathrm{Hz})$ for clamped-clamped functionally graded thick cylinders $(R=0.2 \mathrm{~m}$, $\left.L=2 \mathrm{~m}, S=3, E_{o}=400 \mathrm{GPa}, v_{o}=0.28, \rho_{o}=19300 \mathrm{~kg} / \mathrm{m}^{3}, E_{i}=110 \mathrm{GPa}, v_{i}=0.34, \rho_{i}=8960 \mathrm{~kg} / \mathrm{m}^{3}\right)$

\begin{tabular}{ccccccc}
\hline & \multicolumn{3}{c}{ First bending mode $(m=1, n=1)$} & \multicolumn{2}{c}{ Torsion mode $(m=0, n=1)$} \\
\cline { 2 - 6 }$H / R$ & $\begin{array}{c}\text { Taghvaeipour } \\
\text { et al. }(2012) \\
\left(30^{\mathrm{a}}-744^{\mathrm{b}}\right)\end{array}$ & $\begin{array}{c}\text { Present } \\
(6-117)\end{array}$ & $\begin{array}{c}\text { ANSYS } \\
(3000-3410)\end{array}$ & $\begin{array}{c}\text { Taghvaeipour } \\
\text { et al. }(2012) \\
(30-744)\end{array}$ & $\begin{array}{c}\text { Present } \\
(3-63)\end{array}$ & $\begin{array}{c}\text { ANSYS } \\
(3000-3410)\end{array}$ \\
\hline 0.2 & 369 & 357 & 357 & 639 & 624 & 622 \\
0.4 & 391 & 381 & 379 & 646 & 631 & 629 \\
0.6 & 413 & 401 & 400 & 652 & 636 & 634 \\
0.8 & 434 & 420 & 418 & 656 & 641 & 639 \\
1 & 453 & 437 & 436 & 660 & 644 & 642 \\
1.2 & 470 & 453 & 452 & 662 & 647 & 645 \\
1.4 & 486 & 467 & 462 & 665 & 649 & 647 \\
1.6 & 500 & 479 & 477 & 667 & 651 & 649 \\
1.8 & 513 & 490 & 487 & 668 & 652 & 650 \\
2 & 525 & 500 & 497 & 670 & 654 & 652 \\
\hline
\end{tabular}

${ }^{\mathrm{a}}$ number of elements ${ }^{\mathrm{b}}$ number of degrees of freedom

Table 3. Mechanical properties of silicon nitride and stainless steel

\begin{tabular}{cccc}
\hline \multirow{2}{*}{ Material } & \multicolumn{3}{c}{ Properties } \\
\cline { 2 - 4 } & $E\left(\mathrm{~N} / \mathrm{m}^{2}\right)$ & $v$ & $\rho\left(\mathrm{kg} / \mathrm{m}^{3}\right)$ \\
\hline Silicon nitride $\left(\mathrm{Si}_{3} \mathrm{~N}_{4}\right)$ & $322.27 \times 10^{9}$ & 0.24 & 2370 \\
Stainless steel $(\mathrm{SUS304})$ & $207.78 \times 10^{9}$ & 0.3177 & 8166 \\
\hline
\end{tabular}

A sample convergence study is provided for a cylinder with small and large H/R ratios in Table 4. 5 radial and 10 axial harmonic elements for the thinwalled cylinders seem to be sufficient to achieve a good accuracy whereas 10 radial 10 axial harmonic elements are required for thick-walled cylinders. The number of elements is doubled for satisfactory results for the FGM cylinder of the largest length to radius ratio considered in the study.

The effects of constituent volume fraction and circumferential harmonic number on the natural frequencies of FGM cylinders are studied by varying the value of power law exponent, $S$, between 0.01 and 50 for each circumferential harmonic number from 1 to 10 . Tables 5 and 6 show the variations of the natural frequencies of a thinwalled Model1 and a thick-walled Modell cylinder, respectively. The natural frequencies of the thin- walled Model 2 cylinder decrease with increasing circumferential harmonic number, $m$ until a minimum is reached whereupon they increase as seen in Fig. 3. It seems to be a very typical behavior for isotropic thin cylindrical shells. On the other hand, the thick-walled cylinder behaves in a different way such that the natural frequencies increase almost linearly with increasing circumferential harmonic number as shown in Fig. 4. The natural frequencies approach to those of isotropic cylinder made of stainless steel when the value of power law exponent of Model 1 comes closer to zero. It can be easily concluded that frequency characteristics of functionally graded cylinders are similar to those of isotropic cylinders when the natural frequencies of isotropic stainlesssteel cylinders $(S=0)$ are investigated. 
Table 4. Convergence study for the natural frequencies of thin and thick FG cylinders ( $m=1, R=0.2 \mathrm{~m}, L / R=10, S=10$, Model 1)

\begin{tabular}{cllllllll}
\hline & \multicolumn{7}{l}{$H / R=0.001$} & \multicolumn{7}{l}{$H / R=2.0$} \\
\cline { 2 - 9 } Mesh & $1^{\mathrm{a}}$ & 5 & 10 & 15 & $1^{\mathrm{a}}$ & 5 & 10 & 15 \\
\hline $2^{\mathrm{b}}$ & 529.57 & 525.53 & 525.52 & 525.52 & 892.43 & 875.44 & 875.41 & 875.41 \\
4 & 517.51 & 516.75 & 513.55 & 513.54 & 882.21 & 865.48 & 865.45 & 865.45 \\
6 & 516.88 & 512.93 & 512.92 & 512.92 & 881.58 & 864.87 & 864.84 & 864.84 \\
8 & 516.78 & 512.83 & 512.83 & 512.83 & 881.47 & 864.76 & 864.74 & 864.74 \\
10 & 516.75 & 512.80 & 512.80 & 512.80 & 881.44 & 864.74 & 864.71 & 864.71 \\
12 & 516.75 & 512.80 & 512.80 & 512.80 & 881.43 & 864.73 & 864.70 & 864.70 \\
\hline
\end{tabular}

${ }^{\mathrm{a}}$ Radial mesh size ${ }^{\mathrm{b}}$ Axial mesh size

Table 5. Variation of natural frequencies $(\mathrm{Hz})$ with circumferential harmonic number $m$ and power law exponent $S$ $(H / R=0.005, L / R=10, H=0.005 \mathrm{~m}$, Model 1)

\begin{tabular}{ccccccccc}
\hline$m$ & $S=0$ & $S=0.01$ & $S=0.1$ & $S=0.5$ & $S=1$ & $S=10$ & $S=25$ & $S=50$ \\
\hline 1 & 49.807 & 50.117 & 52.760 & 62.062 & 70.127 & 102.70 & 109.64 & 112.60 \\
2 & 17.590 & 17.702 & 18.653 & 21.977 & 24.830 & 36.207 & 38.619 & 39.650 \\
3 & 12.466 & 12.551 & 13.263 & 15.617 & 17.543 & 25.203 & 26.941 & 27.706 \\
4 & 18.431 & 18.557 & 19.597 & 22.932 & 25.602 & 36.611 & 39.296 & 40.504 \\
5 & 28.891 & 29.087 & 30.697 & 35.836 & 39.953 & 57.176 & 61.441 & 63.366 \\
6 & 42.143 & 42.427 & 44.762 & 52.215 & 58.194 & 83.326 & 89.574 & 92.396 \\
7 & 57.906 & 58.294 & 61.496 & 71.712 & 79.912 & 114.46 & 123.06 & 126.94 \\
8 & 76.121 & 76.631 & 80.834 & 94.249 & 105.02 & 150.44 & 161.75 & 166.86 \\
9 & 96.772 & 97.420 & 102.76 & 119.80 & 133.49 & 191.25 & 205.63 & 212.13 \\
10 & 119.85 & 120.66 & 127.27 & 148.37 & 165.32 & 236.85 & 254.67 & 262.73 \\
\hline
\end{tabular}

Table 6. Variation of natural frequencies $(\mathrm{Hz})$ with circumferential harmonic number $m$ and power law exponent $S$ $(H / R=1.0, L / R=10, H=0.2 \mathrm{~m}$, Model 1)

\begin{tabular}{ccccccccc}
\hline$m$ & $S=0$ & $S=0.01$ & $S=0.1$ & $S=0.5$ & $S=1$ & $S=10$ & $S=25$ & $S=50$ \\
\hline 1 & 352.82 & 354.51 & 368.96 & 421.12 & 468.71 & 700.19 & 762.28 & 789.85 \\
2 & 1277.0 & 1285.9 & 1360.4 & 1602.9 & 1800.2 & 2585.1 & 2762.6 & 2837.9 \\
3 & 2917.7 & 2937.9 & 3106.2 & 3664.0 & 4124.1 & 5948.8 & 6363.5 & 6539.8 \\
4 & 4633.2 & 4663.4 & 4915.9 & 5756.7 & 6456.0 & 9387.1 & 10112 & 10426 \\
5 & 6304.6 & 6341.8 & 6652.3 & 7681.6 & 8548.1 & 12560 & 13684 & 14185 \\
6 & 7876.9 & 7917.2 & 8251.6 & 9361.6 & 10321 & 15313 & 16912 & 17655 \\
7 & 9329.1 & 9369.1 & 9700.6 & 10824 & 11835 & 17661 & 19755 & 20777 \\
8 & 10674 & 10712 & 11029 & 12145 & 13194 & 19725 & 22290 & 23597 \\
9 & 11942 & 11978 & 12283 & 13390 & 14469 & 21620 & 24625 & 26213 \\
10 & 13166 & 13200 & 13495 & 14599 & 15703 & 23416 & 26844 & 28706 \\
\hline
\end{tabular}


Table 7. Variation of fundamental natural frequencies $(\mathrm{Hz})$ with $L / R$ ratios and power law exponent $S(H / R=0.005$, $H=0.005 \mathrm{~m}$, Model 1)

\begin{tabular}{cccccccccc}
\hline$L / R$ & $S=0$ & $S=0.01$ & $S=0.1$ & $S=0.5$ & $S=1$ & $S=10$ & $S=25$ & $S=50$ & $m$ \\
\hline 0.2 & 693.08 & 697.80 & 737.30 & 868.48 & 975.97 & 1402.9 & 1499.3 & 1541.7 & 13 \\
0.5 & 275.70 & 277.57 & 293.26 & 345.57 & 388.54 & 558.96 & 597.18 & 613.94 & 11 \\
1 & 138.02 & 138.95 & 146.78 & 173.06 & 194.80 & 280.22 & 299.80 & 308.10 & 8 \\
2 & 67.790 & 68.248 & 72.099 & 84.991 & 95.627 & 137.73 & 147.09 & 151.18 & 6 \\
5 & 26.094 & 26.271 & 27.756 & 32.708 & 36.779 & 52.921 & 56.537 & 58.121 & 4 \\
10 & 12.465 & 12.550 & 13.261 & 15.616 & 17.541 & 25.200 & 26.938 & 27.703 & 3 \\
20 & 5.4951 & 5.5318 & 5.8422 & 6.8894 & 7.7589 & 11.1965 & 11.953 & 12.282 & 2 \\
50 & 2.2344 & 2.2483 & 2.3659 & 2.7816 & 3.1412 & 4.5957 & 4.9057 & 5.0378 & 1 \\
100 & 0.5615 & 0.5641 & 0.5940 & 0.6980 & 0.7873 & 1.1532 & 1.2321 & 1.2651 & 1 \\
\hline
\end{tabular}
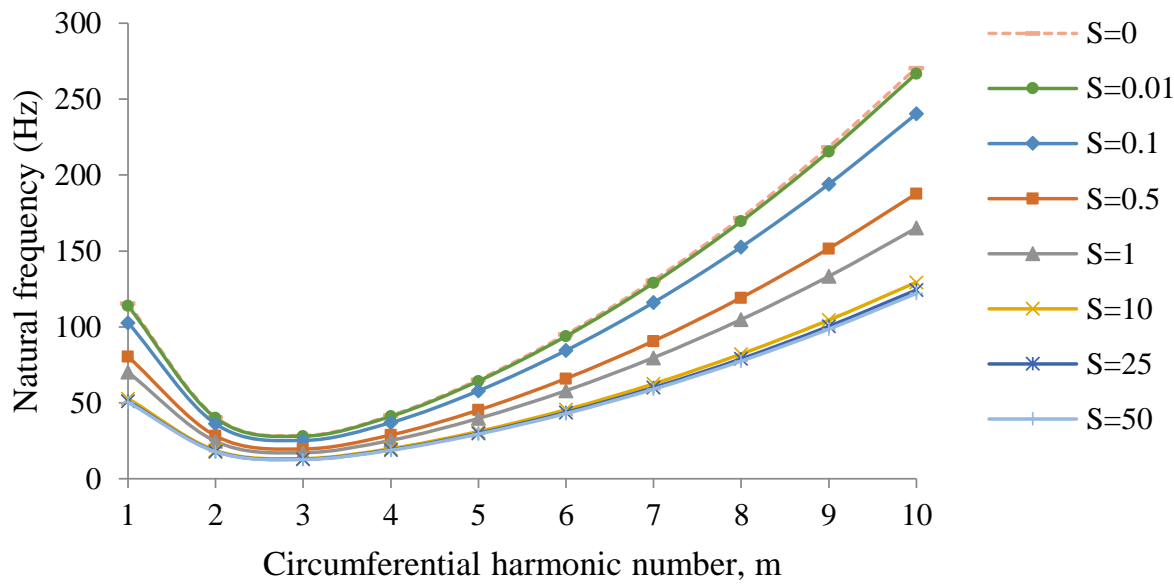

Fig. 3. Variation of natural frequencies $(\mathrm{Hz})$ with circumferential harmonic number $m$ and power law exponent $S(n=1, H / R=0.005, L / R=10, H=0.005 \mathrm{~m}$, Model 2)

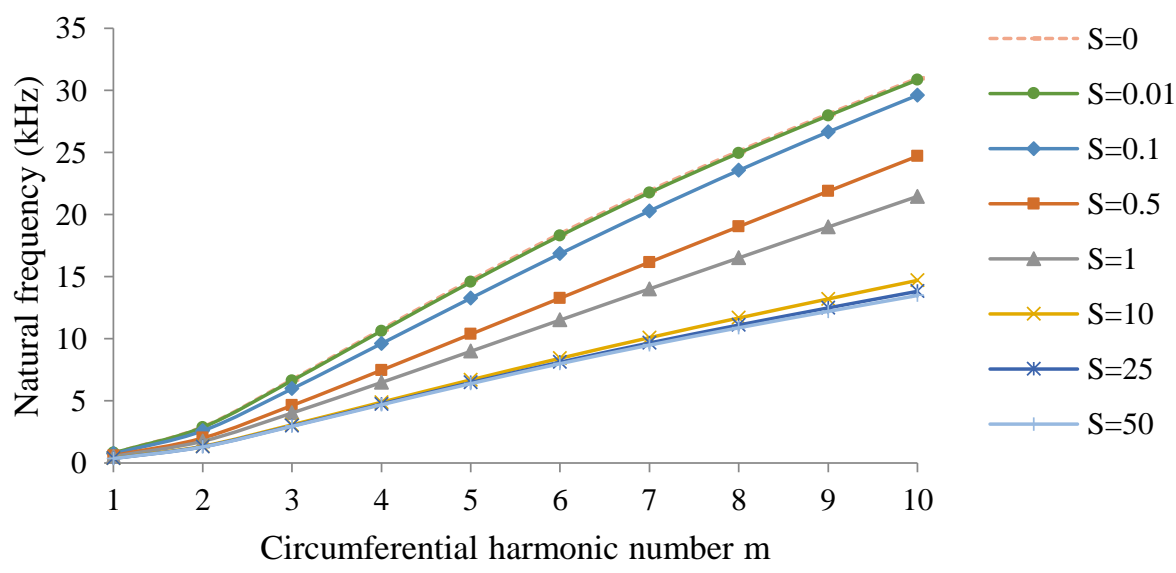

Fig. 4. Variation of natural frequencies $(\mathrm{kHz})$ with circumferential harmonic number $m$ and power law exponent $S(\mathrm{n}=1, \mathrm{H} / \mathrm{R}=1.0, \mathrm{~L} / \mathrm{R}=10, \mathrm{H}=0.2 \mathrm{~m}$, Model2) 
Also, the natural frequencies of Model 1 increase as $S$ increases as it can be seen from Tables 5 and 6 . The influence of $S$ on the natural frequencies of Model 2 is the opposite of Model 1 as seen in Figs. 3 and 4 . Unlike Model 1 cylinder where the natural frequencies increase with increasing $S$, the natural frequencies for Model 2 decrease with increasing $S$. Additionally, the natural frequencies of Model 2 are higher than those of Model 1 for $S<1$ whereas for $S>1$ the frequencies of Model 1 are higher than those of Model 2 for both thin and thick walled functionally graded cylinders.

The percent of differences between natural frequencies obtained at minimum value of $S=0.01$ and maximum value of $S=50$ with various circumferential harmonic numbers for the thin and thick walled Modell cylinders are plotted in Fig. 5.
The value of the power law exponent $S$ does not affect the circumferential harmonic number at which the fundamental natural frequency occurs. In other words, for each $L / R$ ratio corresponding to all power law exponents the fundamental natural frequencies occur at the same circumferential harmonic number $m$, as given in Tables 7 and 8 . Table 7 indicates that the circumferential harmonic number at which the fundamental natural frequency takes place decreases as the thin walled cylinder becomes longer and it becomes one after a particular $L / R$ ratio. On the other hand, Table 8 shows that the fundamental natural frequencies occur at the same circumferential harmonic number $m=1$ for all $L / R$ ratios of thick-walled cylinder with $H / R=1.0$.

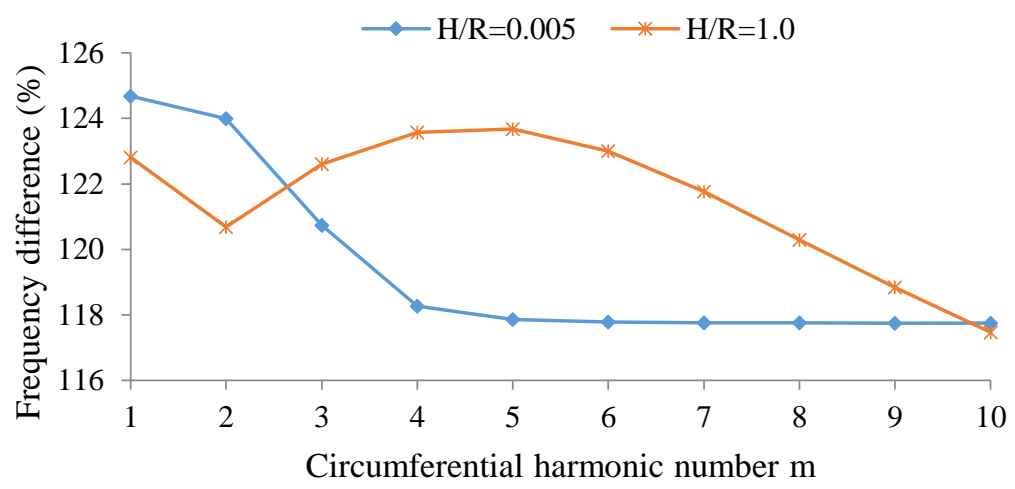

Fig. 5. Differences in natural frequencies of $S=0.01$ and $S=50$ for thin $(H / R=0.005)$ and thick $(H / R=1.0)$ walled Modell cylinders

Table 8. Variation of fundamental natural frequencies $(\mathrm{Hz})$ with $L / R$ ratios and power law exponent $S(H / R=1.0, H=0.2$ m, Model1)

\begin{tabular}{cccccccccc}
\hline$L / R$ & $S=0$ & $S=0.01$ & $S=0.1$ & $S=0.5$ & $S=1$ & $S=10$ & $S=25$ & $S=50$ & $m$ \\
\hline 0.2 & 36492 & 36538 & 36894 & 40639 & 42483 & 55673 & 66229 & 74717 & 1 \\
0.5 & 14038 & 14112 & 14647 & 16067 & 17293 & 25573 & 29219 & 31184 & 1 \\
1 & 6489.9 & 6530.1 & 6865.5 & 7960.3 & 8849.1 & 12948 & 14092 & 14624 & 1 \\
2 & 1674.8 & 1685.3 & 1773.8 & 2074.2 & 2327.9 & 3447.4 & 6465.3 & 3862.7 & 1 \\
5 & 996.15 & 1001.3 & 1045.1 & 1199.0 & 1335.4 & 1985.3 & 2160.5 & 2241.6 & 1 \\
10 & 352.81 & 354.49 & 368.94 & 421.10 & 468.69 & 700.17 & 762.25 & 790.90 & 1 \\
20 & 103.24 & 103.71 & 107.79 & 122.72 & 136.54 & 204.41 & 222.49 & 230.79 & 1 \\
50 & 17.505 & 17.583 & 18.263 & 20.769 & 23.105 & 34.621 & 37.679 & 39.079 & 1 \\
100 & 4.4162 & 4.4360 & 4.6071 & 5.2383 & 5.8272 & 8.7328 & 9.5039 & 9.8569 & 1 \\
\hline
\end{tabular}


Table 9. Variation of fundamental natural frequencies $(\mathrm{Hz})$ with $H / R$ ratios and power law exponents $S(L / R=10, R=0.2$ m, Model 1)

\begin{tabular}{cccccccccc}
\hline$H / R$ & $S=0$ & $S=0.01$ & $S=0.1$ & $S=0.5$ & $S=1$ & $S=10$ & $S=25$ & $S=50$ & $m$ \\
\hline 0.001 & 29.806 & 30.040 & 31.732 & 37.428 & 42.103 & 60.790 & 64.870 & 66.652 & 4 \\
0.003 & 49.945 & 50.278 & 53.092 & 62.620 & 70.560 & 101.92 & 108.78 & 111.75 & 3 \\
0.005 & 62.332 & 62.757 & 66.313 & 78.086 & 87.713 & 126.01 & 134.70 & 138.53 & 3 \\
0.02 & 108.99 & 109.73 & 115.91 & 136.67 & 153.90 & 222.09 & 237.04 & 243.16 & 2 \\
0.04 & 155.87 & 156.95 & 165.93 & 195.14 & 218.81 & 313.69 & 335.53 & 344.61 & 2 \\
0.06 & 209.07 & 210.53 & 222.58 & 261.30 & 292.41 & 418.32 & 447.98 & 460.40 & 2 \\
0.08 & 256.52 & 258.08 & 271.34 & 318.01 & 358.73 & 526.78 & 563.44 & 578.33 & 1 \\
0.1 & 258.55 & 260.11 & 273.37 & 320.11 & 360.94 & 530.38 & 567.60 & 582.75 & 1 \\
0.14 & 262.64 & 264.19 & 277.47 & 324.34 & 365.42 & 537.66 & 576.00 & 591.66 & 1 \\
0.18 & 266.76 & 268.32 & 281.61 & 328.63 & 369.98 & 545.02 & 584.47 & 600.65 & 1 \\
0.3 & 279.30 & 280.87 & 294.25 & 341.81 & 383.98 & 567.49 & 610.30 & 628.04 & 1 \\
0.5 & 300.53 & 302.12 & 315.73 & 364.42 & 408.06 & 605.68 & 654.06 & 674.42 & 1 \\
0.7 & 321.74 & 323.36 & 337.29 & 387.28 & 432.49 & 643.97 & 697.85 & 720.81 & 1 \\
0.9 & 342.60 & 344.27 & 358.55 & 409.97 & 456.75 & 681.69 & 740.94 & 766.47 & 1 \\
1.2 & 372.73 & 374.47 & 389.36 & 442.98 & 492.10 & 736.22 & 803.21 & 832.46 & 1 \\
1.6 & 410.08 & 411.92 & 427.63 & 484.17 & 536.24 & 803.78 & 880.38 & 914.31 & 1 \\
2 & 443.82 & 445.75 & 462.25 & 521.51 & 576.29 & 864.71 & 950.06 & 988.30 & 1 \\
\hline
\end{tabular}

The natural frequencies of thin and thick walled Model 2 cylinders with small $L / R$ ratios are higher than those of with large $L / R$ ratios as shown in Figs. 6 and 7. Also, it can be seen from these figures that the natural frequencies of the thick walled cylinder $(H / R=1.0)$ are higher than those of the thin-walled cylinder $(H / R=0.005)$. Moreover, the fundamental natural frequencies increase with increasing $S$ for Model1 and decrease with increasing $S$ for Model2 thin and thick cylinders. The fundamental natural frequencies for Model1 and Model 2 cylinders occur at the same circumferential harmonic number.

Table 9 shows the variations in the fundamental natural frequencies with thickness to radius ratios $H / R$ and power law exponent $S$ for Model1. The fundamental natural frequencies increase with increasing $S$ and $H / R$. The circumferential harmonic number $m$ at which the fundamental frequency occurs is observed to decrease until a particular $H / R$ ratio. This means that when $H / R$ is beyond a certain value, which is $H / R=0.08$ in our case, the fundamental natural frequencies would occur at the circumferential harmonic number $m=1$. The variation of the circumferential harmonic number at which the fundamental natural frequency occurs with different $H / R$ ratios can be observed as the slope change in Figs. 8 and 9. The changes in the slope in Fig. 8 indicate that the circumferential harmonic number at which the fundamental frequency occurs changes until $H / R=0.08$. Since there is no slope change for the ratios greater than this ratio as shown in Fig. 9 it is obvious that the fundamental frequencies occur at the same circumferential harmonic number for these ratios. Unlike Model 1, Figs. 8 and 9 show that the fundamental natural frequencies decrease with increasing $S$ for Model 2. 


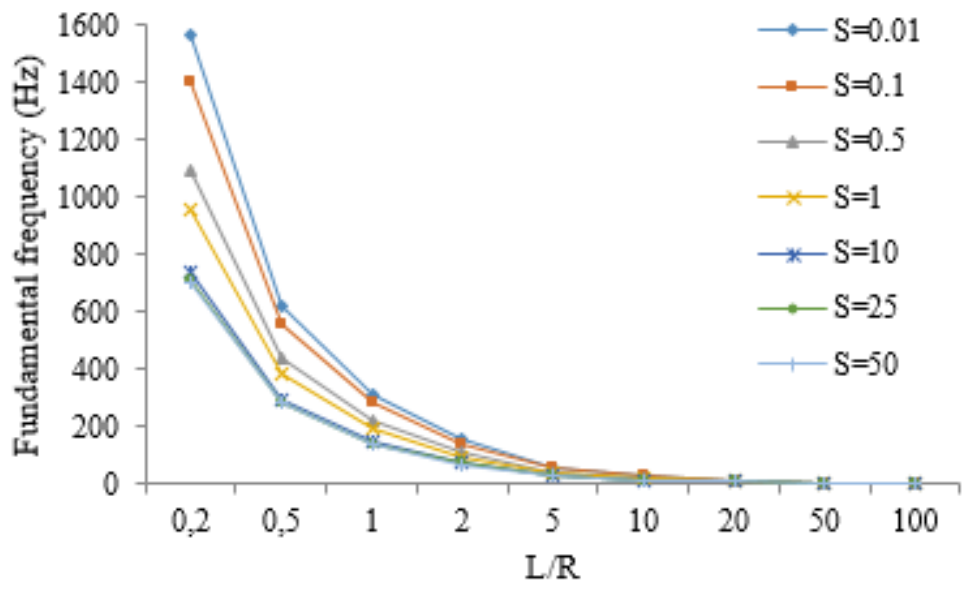

Fig. 6. Variation of fundamental natural frequencies $(\mathrm{Hz})$ with $L / R$ ratios and power law exponent $S(\mathrm{n}=1, H / R=0.005$, $H=0.005 \mathrm{~m}$, Model2)

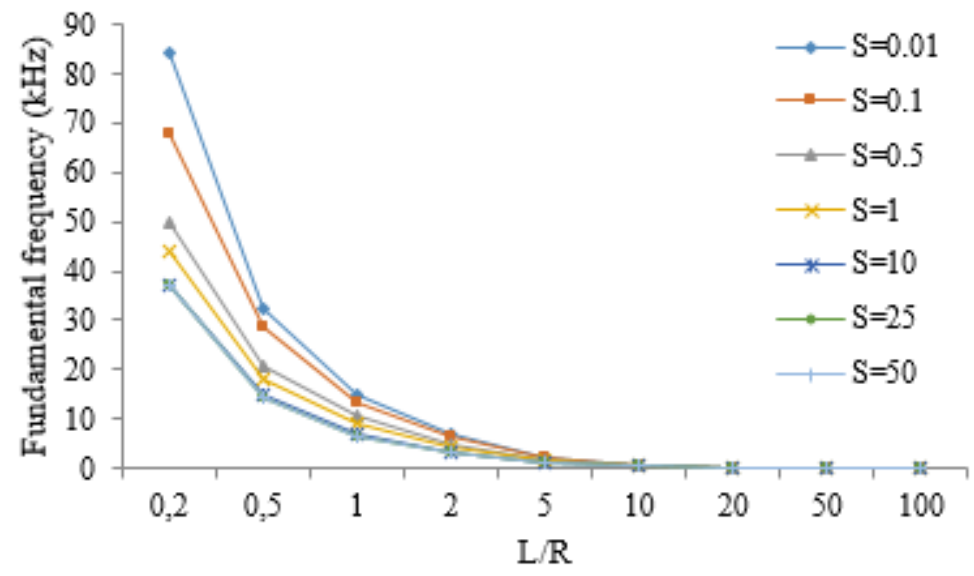

Fig. 7. Variation of fundamental natural frequencies $(\mathrm{kHz})$ with $L / R$ ratios and power law exponents $S(n=1, H / R=1.0$, $H=0.2 \mathrm{~m}$, Model2)

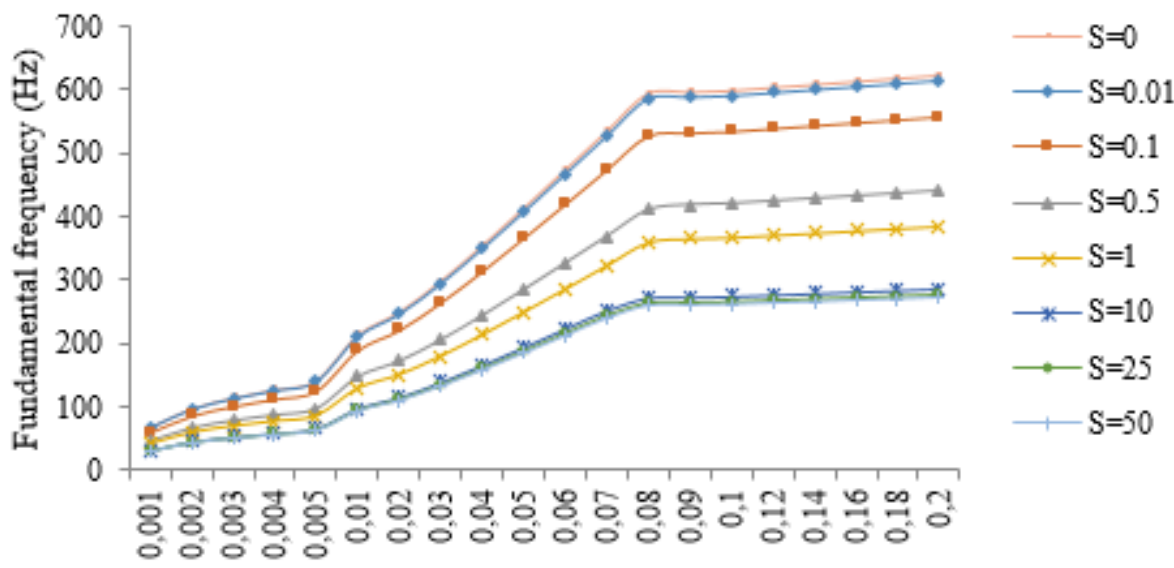

$\mathrm{H} / \mathrm{R}$

Fig. 8. Variation of fundamental natural frequencies $(\mathrm{Hz})$ with $H / R$ ratios and power law exponents $S(n=1 . L / R=10$. $R=0.2 \mathrm{~m}$, Model2) 


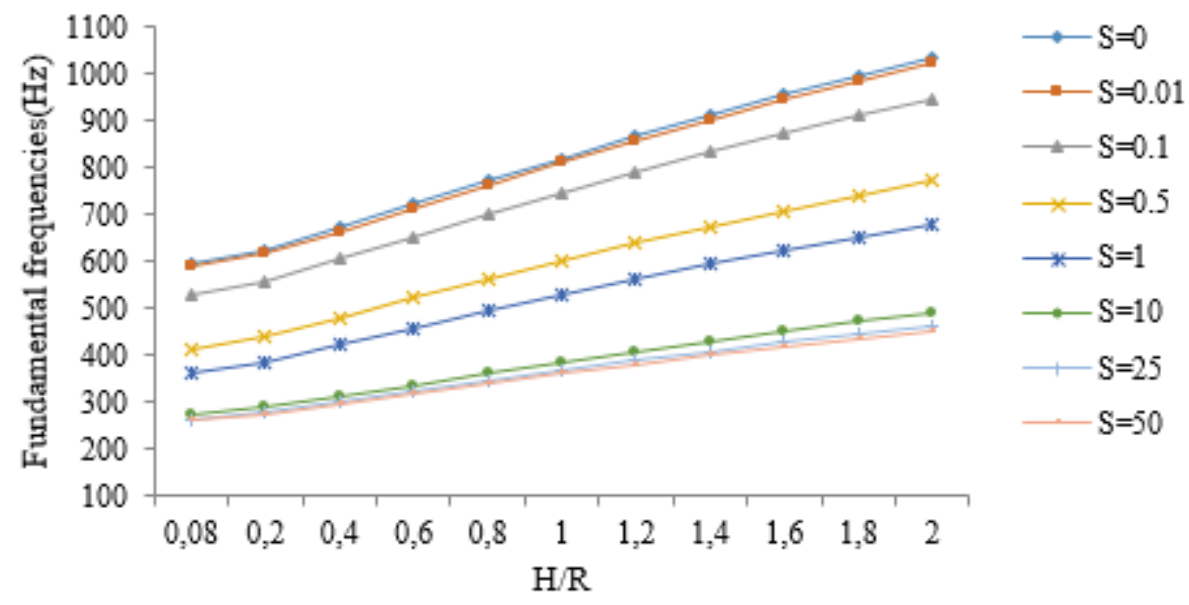

Fig. 9. Variation of fundamental natural frequencies $(\mathrm{Hz})$ with $H / R$ ratios and power law exponents $S(n=1 . L / R=10$. $R=0.2 \mathrm{~m}$, Model 2 )

\section{Conclusions}

In the present study, a graded harmonic solid ring finite element is developed for free vibration analysis of functionally graded hollow thin and thick-walled cylinders. In the element formulation Fourier series expansion is used in the circumferential direction $\theta$, and the threedimensional theory of elasticity is used for the model rather than a shell theory. Detailed parametric studies are carried out to show the influences of the power law exponent $\mathrm{S}$, the constituent material position, the thickness to radius ratio $H / R$, and the length to radius ratio $L / R$ on the free vibration frequencies. The natural frequencies of simply-simply supported thinwalled and clamped-clamped supported thickwalled FG cylinders are compared with those available in the literature for verification. The results that were extracted by the graded harmonic ring finite elements indicate high accuracy and good agreement with those obtained in the previous studies. As far as numerical results are considered the following conclusions can be drawn:

* The frequencies of the thin-walled FGM cylinders decrease with increasing circumferential number until a minimum is reached whereupon they increase while those of the thick-walled cylinders increase almost linearly.
* The influence of the power law exponent on the frequency depends on the constituent material position. Depending on the material position it may lead to an increase or decrease in the frequency.

* The power law exponent does not affect the circumferential harmonic number at which the fundamental natural frequency occurs.

* The circumferential harmonic number at which the fundamental natural frequency takes place decreases as the thin-walled cylinder becomes longer and it becomes $m=1$ after a particular $L / R$ ratio. On the other hand, the fundamental natural frequencies occur at the same circumferential harmonic number $\mathrm{m}=1$ for all $L / R$ ratios of thick-walled cylinder with $H / R=1.0$.

* The fundamental natural frequencies increase with increasing $H / R$ ratio.

* The circumferential harmonic number at which the fundamental frequency occurs is observed to decrease until a particular $H / R$ ratio and beyond this ratio it becomes $m=1$.

\section{Acknowledgements}

The first author has been supported by TUBITAK (Turkish Scientific and Technological Research Council) with a scholarship which is gratefully acknowledged. 


\section{References}

[1] Cho JR and Kim BG (1999) Finite element analysis of thermal stresses in functionally gradient layered composites. KSME International Journal 13: 108115 .

[2] Ludwig A and Krieg R (1981) An analytical quasiexact method for calculating eigen-vibrations of thin circular cylindrical shells. Journal of Sound and Vibration 74: 55-174.

[3] Chung H (1981) Free vibration analysis of circular cylindrical shells. Journal of Sound and Vibration 74: 331-350.

[4] Bhimaraddi A (1984) A higher order theory for free vibration analysis of circular cylindrical shells. International Journal of Solids and Structures 20: 623-630.

[5] Wang H and Williams K (1996) Vibrational modes of thick cylinders of finite length. Journal of Sound and Vibration 191: 955-971.

[6] Loy CT and Lam KY (1999) Vibration of thick cylindrical shells on the basis of three-dimensional theory of elasticity. Journal of Sound and Vibration 226: 719-737.

[7] Singhal RK, Guan W and Williams K (2001) Modal analysis of a thick-walled circular cylinder. Mechanical Systems and Signal Processing 16: 141-153.

[8] Loy CT, Lam KY and Reddy JN (1999) Vibration of functionally graded cylindrical shells. International Journal of Mechanical Sciences 41:309-324.

[9] Pradhan SC, Loy CT, Lam KY and Reddy JN (2000) Vibration characteristics functionally graded cylindrical shells under various boundary conditions. Applied Acoustics, 61: 111-129.

[10] Kadoli R and Ganesan K (2006) Buckling and free vibration analysis of functionally graded cylindrical shells subjected to a temperaturespecified boundary condition. Journal of Sound and Vibration 289: 450-480.

[11] Iqbal Z, Nawaz MN and Sultana N (2009) Vibration characteristics of FGM circular cylindrical shells using wave propagation approach. Acta Mechanica. 208: 237-248.

[12] Arshad SH, Naeem MN, Sultana N, Iqbal Z and Shah AG (2009) Vibration of bi-layered cylindrical shells with layers of different materials. Journal of Mechanical Science and Technology 24: 805-810.

[13] Shah AG, Mahmood T, Naeem MN, Iqbal Z and Arshad SH (2009) Vibrations of functionally graded cylindrical shells based on elastic foundations. Acta Mechanica 211: 293-307.

[14] Naeem MN, Ahmad M, Shah AG, Iqbal N and Arshad SH (2010) Applicability of generalized differential quadrature method for vibration study of FGM cylindrical shells. European Journal of Scientific Resarch 47: 82-99.

[15] Ansari R and Darvizeh M (2008) Prediction of dynamic behavior of FGM shells under arbitrary boundary conditions. Composite Structures 85: 284-292.

[16] Patel BP, Gupta SS, Loknath MS and Kadu CP (2005) Free vibration analysis of functionally graded elliptical cylindrical shells using higherorder theory. Composite Structures 69: 259-270.

[17] Haddapour H, Mahmoudkhani S and Navazi HM (2007) Free vibration analysis of functionally graded cylindrical shells including thermal effects. Thin Walled Structures 45:591-599.

[18] Li Y, Ramesh KT and Chin ESC (2001) Dynamic characterization of layered and graded structures under impulsive loading. International Journal of Solids and Structures 38: 6045-6061.

[19] Anlas G, Santare MH and Lambros J (2000) Numerical calculation of stress intensity factors in functionally graded materials. International Journal of Fracture 104: 131-143.

[20] Santare MH, Thamburaj P and Gazonas GA (2003) The use of graded finite elements in the study of elastic wave propagation in continuously nonhomogeneous materials. International Journal of Solids and Structures 40: 5621-5634.

[21] Kim JH and Paulino GH (2002) Isoparametric graded finite elements for nonhomogeneous isotropic and orthotropic materials. Journal of Applied Mechanics 69: 502-514.

[22] Taghvaeipour A, Bonakdar M and Ahmadian MT (2012) Application of a new cylindrical element formulation in finite element structural analysis of FGM hollow cylinders. Finite Elements in Analysis and Design 50: 1-7.

[23] Darabseh TT (2011) Transient thermal stresses of functionally graded thick hollow cylinder under the green-lindsay model. World Academic Science and Engineering Technology 59: 2314-2318.

[24] Benasciutti D, De Bona F and Munteanu MGH (2011) Numerical Analysis-Theory and Application, In: Awrejcewicz J. (Editor), University of Udine. 71-96. 
[25] Bhatti MA. Advanced Topics in Finite Element Analysis of Structures: with Mathematica and Matlab Computations. USA: John Wiley\&Sons, 2006. 